\title{
A catalogue of ultra-luminous X-ray source coincidences with FIRST radio sources ${ }^{\star}$ (Research Note)
}

\author{
J. R. Sánchez-Sutil ${ }^{1}$, A. J. Muñoz-Arjonilla ${ }^{1}$, J. Martî2,1 , J. L. Garrido ${ }^{2,1}$, \\ D. Pérez-Ramírez ${ }^{2,1}$, and P. Luque-Escamilla ${ }^{3,1}$ \\ 1 Grupo de Investigación FQM-322, Universidad de Jaén, Campus Las Lagunillas s/n, Edif. A3, 23071 Jaén, Spain
2 Departamento de Física, EPS, Universidad de Jaén, Campus Las Lagunillas s/n, Edif. A3, 23071 Jaén, Spain
e-mail: [jmarti; jlg; dperez] @ujaen. es
Dpto. de Ing. Mecánica y Minera, EPS, Universidad de Jaén, Campus Las Lagunillas s/n, Edif. A3, 23071 Jaén, Spain
e-mail: peter@ujaen. es
}

Received 14 December 2005 / Accepted 9 March 2006

\section{ABSTRACT}

\begin{abstract}
Aims. We search for ultra luminous X-ray source (ULXs) radio counterparts located in nearby galaxies in order to constrain their physical nature.

Methods. Our work is based on a systematic cross-identification of the most recent and extensive available ULX catalogues and archival radio data.

Results. A catalogue of 70 positional coincidences is reported. Most of them are located within the galaxy nucleus. Among them, we find 11 new cases of non-nuclear ULX sources with possibly associated radio emission.
\end{abstract}

Key words. X-rays: galaxies - X-ray: stars - radio continuum: galaxies - radio continuum: stars - X-rays: binaries Black hole physics

\section{Introduction}

Ultra luminous X-ray sources (ULXs) are common phenomena nowadays. Detected in several galaxies as extranuclear X-ray sources, their luminosities are often in excess of $\sim 10^{39} \mathrm{erg} \mathrm{s}^{-1}$ in the soft X-ray bands as observed by several satellite observatories (ROSAT, XMM, etc.). Their existence was observationally stablished nearly two decades ago (Fabbiano 1989). Also known as intermediate-luminosity X-ray objects (IXOs), their physical nature still remains controversial. Unless beaming effects were at work, their intrinsic luminosities are apparently in between those of normal X-ray binaries (e.g. $\sim 10^{38} \mathrm{erg} \mathrm{s}^{-1}$ ) and X-ray emitting active galaxies (e.g. $\sim 10^{43} \mathrm{erg} \mathrm{s}^{-1}$ in the M 87 example).

Several hypothesis have been proposed to explain such extraordinary objects, from accreting intermediate mass black holes (IMBH) having $\sim 10^{2}-10^{4} M_{\odot}$ to microquasar X-ray binaries with strong beaming effects (i.e. microblazars). In particular, the IMBH interpretation has been recently favoured, based on X-ray variability studies and spectral fits in the M74 ULX, and supported by the estimated orbital period in M $82 \mathrm{X}-1$, which implies black holes of thousands of solar masses (Liu et al. 2005; Kaaret et al. 2006). The reader is referred to Fabbiano (2004), Miller \& Colbert (2004), Soria et al. (2005), and Fabbiano \& White (2006) for some recent reviews.

Progress towards a solution to the ULX problem has been possible in a few cases for which a candidate radio counterpart was detected. At radio wavelengths, extinction by the interstellar

* Tables 3 and 4 and Figs. 1-9 are only available in electronic form at http: //www. edpsciences.org medium is not an issue in contrast to other domains of the electromagnetic spectrum. Moreover, modern radio interferometers provide excellent angular resolution and sensitivity when searching for faint radio counterparts and studying their spectral index and variability properties. The case of the ULX in NGC 5408 reported by Kaaret et al. (2003) is a prototype example of this approach followed by other radio works (Körding et al. 2005; Miller et al. 2005).

Unfortunately, the cases for which radio counterparts are available are not significant enough to establish statistically robust conclusions. Therefore, efforts to increase the number of radio detections could potentially lead to further evidence.

The total number of ULX objects has been growing extensively in past years. In particular, two extensive catalogues of ULXs have recently become available from Liu \& Mirabel (2005) and Liu \& Bregman (2005), containing several hundreds of these objects. This fact, together with the availability of sensitive radio surveys at high galactic latitudes, makes it feasible and worthwhile to attempt a systematic crossidentification search for new cases of ULX with associated radio emission. The Faint Images of the Radio Sky (FIRST) survey carried out with the Very Large Array (VLA) at $20 \mathrm{~cm}$ becomes specially suitable for such a purpose given both its good sensitivity ( $\mathrm{rms} \sim 0.15 \mathrm{mJy}$ ) and angular resolution $\left(\sim 5^{\prime \prime}\right)$, together with its associated catalogue compiled by White et al. (1997).

In this context, our goal in the present paper is to present the results of a search based on a systematic cross-identification of the whole $\sim 8 \times 10^{5}$ FIRST radio sources in the most recent catalogues of the ULX sources quoted above. This approach is similar to the one carried out by Miller \& Mushotzky that 
Table 1. List of ULX catalogues cross-identified with FIRST.

\begin{tabular}{lccc}
\hline \hline catalogue & $\begin{array}{c}\text { Number of } \\
\text { galaxies }\end{array}$ & $\begin{array}{c}\text { Number of } \\
\text { ULX }\end{array}$ & $\begin{array}{c}\text { Desig- } \\
\text { nation }\end{array}$ \\
\hline Colbert \& Ptak (2002) & 54 & 87 & CP \\
Liu \& Mirabel (2005) & 85 & 229 & LM \\
Liu \& Bregman (2005) & 313 & 562 & LB \\
\hline
\end{tabular}

covered nearby galaxies observed by Chandra and XMM as cited in Mushotzky (2004). In our case, several new positional coincidences of FIRST radio sources with ULXs have been found. They did not appear to be previously recognized as such in the literature according to the SIMBAD database. The following sections are devoted to describing the cross-identification method and presenting the results. Both radio maps and individual discussion of coincidence cases of special interest are also included.

\section{Cross-identification method and results}

The ULX catalogues used in this work are listed in Table 1. Among them, the most extensive one is that by Liu \& Bregman (2005), which became the main reference for our search as it includes most entries. Our search is naturally restricted to the galaxies in the FIRST radio survey, which implies, for instance, that only $46 \%$ of the Liu \& Bregman (2005) ULXs are covered.

Our cross-identification was based on the positional coincidence of the FIRST and ULX entries within less than 10". This value comes from the typical positional uncertainty of the ROSAT data on the X-ray catalogues. The FIRST positional accuracy is around or better than $1^{\prime \prime}$, i.e., a value that is negligible compared to that of ROSAT. The cross-identification was carried out in practice by means of a fortran code that reads and compares the FIRST and ULXs catalogues previously downloaded from electronic public archives.

A total of 70 matches resulted from the crossidentification, which represents $27 \%$ of the ULX sources in the Liu \& Bregman (2005) reference catalogue covered by FIRST. We list all of them in Table 3 in electronic format, where the remarks column indicates coincidences with the galaxy nucleus, extended radio emission, and previous identifications in the SIMBAD database. At the FIRST angular resolution, the expected appearance of a ULX radio counterpart is that of a compact source for most plausible ULX physical scenarios (IMBH, microblazar, etc.). Nevertheless, several extended nebulae have been recently reported around some ULX sources (Pakull et al. 2003; Kaaret et al. 2004; Miller et al. 2005), which would be better explained in a scenario where an IMBH isotropically excites a bubble nebulae around the ULX. Hence, coincidences with extended radio sources were finally kept (17\% of matches).

Most entries in Table $3(80 \%)$ overlap with the galaxy nucleus and, therefore, they are probably not true ULX sources but simply X-ray emission from the galaxy's central black hole. Among non-nuclear matches, only a few that are identified in the SIMBAD database are up to date. The remaining 11 coincidences appear as the interesting sources to be reported here as they represent new radio counterpart candidates of non-nuclear ULX sources (see Table 2). The nature of these objects is tentatively suggested based on their location and morphology. Thus, a QSO candidate is considered for compact or double radio sources at a peripheral galactic location. On the other hand, coincidences of extended radio emission within knotty optical features or spiral arms are taken as likely HII regions.

For each of these selected sources, we also show electronically in Figs. 1 to 8 the optical and radio fields to illustrate their positional coincidence. The optical images were retrieved from the Digitized Sky Survey (DSS) (Lasker et al. 1990), while the radio ones were taken from FIRST. The following section is devoted to providing a more detailed individual discussion. It has been necessary to adopt a distance for the different studied galaxies as indicated in the electronic Table 4 . These values were computed based on the Hubble law and assuming a Hubble constant of $H_{0}=75 \mathrm{~km} \mathrm{~s}^{-1} \mathrm{Mpc}^{-1}$.

\section{Comments on selected non-nuclear coincidences}

$X 1$ in NGC 3738. The Sd irregular galaxy NGC 3738 is a member of a non interacting pair. The FIRST survey shows a double radio source at nearly two arc-minutes from the galaxy nucleus. Only one radio component is consistent with the ROSAT HRI position of the ULX source (see electronic Fig. 1). As mentioned above, ULX radio counterparts are likely to be compact objects in the FIRST images. Therefore, the detection of a double radio source strongly suggests that X1 in NGC 3738 is not a ULX in this galaxy but is an unrelated background object, possibly a QSO. This suggestion is further supported by both its strong radio flux density, which outshines the galaxy itself, and the ULX's very peripheral location.

X1 in NGC 4088. The Holmberg SBc galaxy NGC 4088 forms a pair with NGC 4085 with both belonging to the Ursa Major Cluster. The ULX source is within the extended emission of a spiral arm in NGC 4088 as shown in electronic Fig. 2. Nevertheless, the X-ray position is coincident with a conspicuous maximum of radio emission. In spite of this fact and based on the available data, we cannot rule out that most of the radio emission comes from an HII region in the spiral arm.

X6 in NGC 4258. The nearby and almost edge-on barred (SAB(s)bc) galaxy NGC 4258 (Messier 106) hosts a weak AGN. It also forms a pair with NGC 4248 in the Ursa Major Cluster. The optical image (see electronic Fig. 3) shows an apparent chain of HII regions at the extremities of its arms. This galaxy is well known for its nuclear thin disk in Keplerian rotation, which is traced by emission from water masers (Miyoshi et al. 1995) and implies a supermassive central black hole.

This case is reminiscent of the first object discussed above, both in flux density level and galactocentric distance. The radio morphology suggests a core+jet structure with the brighter component being consistent with the X-ray position. Again, we speculate that this object is most likely a background QSO unrelated to the galaxy.

X2 in NGC 4395. The galaxy NGC 4395 has a loose and disconnected appearance containing the faintest and nearest Seyfert 1 nucleus currently known and classified as Sc/Irr type. The proposed counterpart of X2 in NGC 4395 is a very compact and faint radio source in the peripheral outskirts of the galaxy (electronic Fig. 4). With the present data it is difficult to assess whether the object is associated to NGC 4395 itself. 
Table 2. Selected non-nuclear, compact, and unidentified candidate radio counterparts of ULX sources.

\begin{tabular}{|c|c|c|c|c|c|c|c|c|}
\hline$\overline{\text { NGC }}$ & $\begin{array}{l}\text { ULX } \\
\text { Name }\end{array}$ & $\begin{array}{c}\alpha_{\text {J2000.0 }} \\
\text { (FIRST) }\end{array}$ & $\begin{array}{c}\delta_{\mathrm{J} 2000.0} \\
(\mathrm{FIRST})\end{array}$ & $\begin{array}{c}\text { Radio vs. } \\
\text { X-ray } \\
\text { offset }\end{array}$ & $\begin{array}{c}\text { Peak } \\
\text { flux density } \\
(\mathrm{mJy})\end{array}$ & $\begin{array}{l}\text { Integrated } \\
\text { flux density } \\
(\mathrm{mJy})\end{array}$ & $\begin{array}{l}\text { Procedence } \\
\text { catalogue(s) }\end{array}$ & Comments \\
\hline 3738 & $\mathrm{X} 1$ & $11^{\mathrm{h}} 35^{\mathrm{m}} 45^{\mathrm{s}} \cdot 42$ & $+54^{\circ} 33^{\prime} 15^{\prime \prime}{ }^{\prime} 4$ & 0.06 & 61.37 & 94.11 & LB & QSO? \\
\hline 4088 & $\mathrm{X} 1$ & $12^{\mathrm{h}} 05^{\mathrm{m}} 31^{\mathrm{s}} \cdot 70$ & $+50^{\circ} 32^{\prime} 46^{\prime \prime} 8$ & 3." 62 & 1.87 & 5.07 & $\mathrm{LB}, \mathrm{LM}, \mathrm{CP}$ & HII region? \\
\hline 4258 & X6 & $12^{\mathrm{h}} 18^{\mathrm{m}} 46^{\mathrm{s}} \cdot 32$ & $+47^{\circ} 14^{\prime} 20^{\prime \prime} 4$ & 3." 90 & 7.14 & 12.44 & LB & QSO? \\
\hline 4395 & $\mathrm{X} 2$ & $12^{\mathrm{h}} 25^{\mathrm{m}} 32^{\mathrm{s}} \cdot 31$ & $+33^{\circ} 25^{\prime} 34^{\prime \prime} 0$ & $0 .{ }^{\prime \prime} 05$ & 1.35 & 1.28 & LB & - \\
\hline 4449 & $\mathrm{X} 4$ & $12^{\mathrm{h}} 28^{\mathrm{m}} 10^{\mathrm{s}} \cdot 96$ & $+44^{\circ} 06^{\prime} 48^{\prime \prime} 4$ & 3." 30 & 9.27 & 9.81 & LB & SNR? \\
\hline 4559 & $\mathrm{X} 4$ & $12^{\mathrm{h}} 35^{\mathrm{m}} 56^{\mathrm{s}} .30$ & $+27^{\circ} 59^{\prime} 26^{\prime \prime} 4$ & 5." 35 & 1.12 & 0.90 & LB & HII region? \\
\hline 4861 & $\mathrm{X} 2$ & $12^{\mathrm{h}} 59^{\mathrm{m}} 00^{\mathrm{s}} \cdot 35$ & $+34^{\circ} 50^{\prime} 42^{\prime \prime} 9$ & $1 . " 97$ & 3.88 & 6.01 & $\mathrm{LB}, \mathrm{LM}, \mathrm{CP}$ & HII region \\
\hline 5457 & X9 & $14^{\mathrm{h}} 03^{\mathrm{m}} 41^{\mathrm{s}} \cdot 42$ & $+54^{\circ} 19^{\prime} 05^{\prime \prime} 2$ & 3." 40 & 6.63 & 11.45 & LB, LM & HII region? \\
\hline 5457 & X17 & $14^{\mathrm{h}} 02^{\mathrm{m}} 28^{\mathrm{s}} \cdot 12$ & $+54^{\circ} 16^{\prime} 27^{\prime \prime} 3$ & 4." 30 & 1.10 & 1.97 & LB, LM & HII region? \\
\hline 5457 & X26 & $14^{\mathrm{h}} 04^{\mathrm{m}} 29^{\mathrm{s}} .14$ & $+54^{\circ} 23^{\prime} 53^{\prime \prime}{ }^{\prime \prime} 4$ & 4."07 & 2.65 & 4.05 & LB, LM & HNR? SSS? \\
\hline 5457 & X29 & $14^{\mathrm{h}} 04^{\mathrm{m}} 00^{\mathrm{s}} .78$ & $+54^{\circ} 09^{\prime} 11^{\prime \prime} 3$ & 6." 36 & 3.68 & 5.43 & LB & FRII? \\
\hline
\end{tabular}

X4 in NGC 4449. The very blue Magellanic irregular galaxy NGC 4449 contains a lot of HII regions. In this case, the ULX radio counterpart candidate is a strictly compact radio source projected against the galactic disk (electronic Fig. 5).

In the FIRST image, it outshines the galactic nucleus. Thus, the possibility that we are again dealing with a background object cannot be ruled out. Alternatively, the ULX source could be associated with some of the apparent extended regions seen at optical wavelengths within the X-ray error box. In fact, this ULX source corresponds to source 15 in the Chandra results reported by Summers et al. (2003), who assumed it to be a supernova remnant (SNR) based on the old VLA observations of Bignell \& Seaquist (1983) with angular resolution poorer than FIRST.

X4 in NGC 4559. The Sc spiral galaxy NGC 4559 exhibits some easily resolved HII regions. X4 coincides with a compact and faint radio counterpart candidate in the FIRST image as illustrated in electronic Fig. 6. It is clearly located within one of the spiral arms. At optical wavelengths there is a possible HII region near the ULX position that could be associated to. However, no extended radio emission is detected around it.

X2 in NGC 4861. The galaxy NGC 4861 is part of the Mrk 59 complex, together with the dwarf irregular galaxy IC 3961. In particular, NGC 4861 refers to the bright knot in the southern region of the complex (see electronic Fig. 7). It has been suggested that it is an HII region powered by massive early type stars. The proposed radio counterpart is a barely extended radio source very close to it.

X9, X17, X26, and X29 in NGC 5457. Also known as Messier 101, NGC 5457 is a nearby face-on Sc spiral galaxy within a rich group. It is the prototype of the multiple-arm galaxies of the Sc classification.

X9 and X17 appear projected against the spiral arms of the galactic disk (see electronic Fig. 8). Extended emission around their proposed radio counterparts is clearly detected. Therefore, the association with these regions of star formation cannot be ruled out in these cases. In contrast, X26 and X29 in Messier 101 have radio counterpart candidates with apparently double morphology (see again Fig. 8). They are located in the peripheral region of the galaxy, so we could also be dealing with background sources as in the first ULX case discussed in this section. In particular, X29 reminds of a Fanaroff-Riley Type II radio galaxy. On the other hand, X26 seems to overlap with a fuzzy optical object (NGC 5471B) that has been proposed by Jenkins et al. (2004) to be either an hypernova remnant (HNR) or a supersoft source (SSS) of X-ray binary type.

\section{Discussion}

We notice that 6 out of the 11 selected sources in Table 2 appear to overlap with spiral arms or HII regions in their corresponding galaxy. This fact is not new (Fabbiano 2004; Liu \& Bregman 2001) and could be an indication of ULX sources forming predominantly in star formation regions. Such a location would support the idea of ULX sources being related to end products of fast evolving massive stars. Thus, if IMBH exist, some of them could be linked to the final stages of very high mass stars (Madau $\&$ Rees 2001). On the other hand, it also cannot be ruled out that some of our objects are merely background AGNs or QSOs and, therefore, they are related to supermassive black holes. The number of wrong identifications in Liu \& Bregman (2005) can be tentatively estimated by extrapolating our sample over the entire catalogue. Given that Table 3 contains at least three possible QSOs, this would imply a minimum of 24 QSOs falsely identified as ULXs. More realistically, this number could represent more than half of the total catalogue entries if radio-loud nuclear coincidences were also considered as non-secure identifications.

A possible way to try to constrain the nature of sources in Table 2 is to study how they behave with respect to the correlation between radio $\left(L_{\text {radio }}\right)$ and X-ray $\left(L_{\mathrm{X}}\right)$ luminosity and black hole mass $(M)$ proposed by several authors for black holes with sub-Eddington accretion rates. Such a correlation appears to be valid for stellar, supermassive, and intermediate mass black holes, and both observational and theoretical evidence seems to support it (see e.g. Körding et al. 2002; Corbel et al. 2003; Gallo et al. 2003; Merloni et al. 2003; Falcke et al. 2004 and references therein). In these systems, the spectral energy distribution is dominated by the non-thermal emission from a relativistic jet. The spectrum can be approximated by a broken power law with a flat-to-inverted optically thick part, typically in the radio domain, and with a steep optically thin part at X-ray energies. In this context ULXs would be low-mass analogs of BL Lac objects. Using the Falcke et al. (2004) formulation, the non-linear correlation can be generally expressed as

$L_{\mathrm{X}} \propto L_{R}^{m} M^{\alpha_{\mathrm{X}}-m \alpha_{R}}$

where $m=\left(17 / 12-2 \alpha_{\mathrm{X}} / 3\right) /\left(17 / 12-2 \alpha_{R} / 3\right)$ and, on average, the radio optically thick and $\mathrm{X}$-ray optically thin spectral indexes are 
$\alpha_{R} \simeq 0.15$ and $\alpha_{\mathrm{X}} \simeq-0.6$, respectively. For practical purposes we adopt these values so Eq. (1) then becomes

$L_{\mathrm{X}} \propto L_{R}^{1.38} M^{-0.81}$.

To test the correlation for our ULX sources we have used the distances from electronic Table 4 as quoted before. The X-ray luminosities were taken from Liu \& Bregman (2005), while the monochromatic radio luminosities are those derived from FIRST flux densities at $1.4 \mathrm{GHz}$. The Falcke et al. (2004) correlation parameters were established using the X-ray energy band of $2-10 \mathrm{keV}$ and the monochromatic radio luminosity at $5 \mathrm{GHz}$. For consistency, we have consequently scaled our $L_{\mathrm{X}}$ and $L_{\text {radio }}$ values by using the spectral index values just quoted above, and the resulting plot is shown in electronic Fig. 9.

It can be seen here that none of the selected ULX sources studied would apparently correspond to an IMBH. In the framework of correlations in the sub-Eddington accretion regime, this suggests that most of these objects would be background AGNs unrelated to their respective galaxy, as we previously thought. Independent of the correlation, such a statement is especially likely in cases of double radio sources in NGC 3738, NGC 4258, and NGC 5457. A true ULX nature for some of the other sources is not strictly excluded especially if other physical scenarios are considered, such as strongly beamed stellar sources. Further observations with higher angular resolution and sensitivity will be certainly needed before a new radio emitting ULX source of the one reported in this work could confidently be claimed to be a true ULX.

\section{Conclusions}

We have presented a catalogue of 70 ULX coincidences with FIRST radio sources based on a cross-identification. This constitutes an example of how modern available databases and archives are powerful resources still to be exploited towards solving different issues in today's astrophysics. Most entries are likely nuclear coincidences with their respective galaxy and, therefore, not true ULX in a strict sense. We do find 11 cases of non-nuclear ULXs with new radio counterpart candidates that have got our attention.

Among them, however, most are probably background QSOs or AGNs and not proper ULX sources. Double radio morphology with typical quasar or radio galaxy appearance supports this statement in at least 4 objects. For the rest, the background hypothesis is further suggested based on their behaviour with respect to the correlation between X-ray and radio luminosities and the mass of the black hole accreting at subEddington regimes. In particular, our correlation plot indicates supermassive black holes $\left(\sim 10^{6}-10^{9} M_{\odot}\right)$, and these values are not expected at all in a galaxy disk, given the peripheral location of the sources.

Nevertheless, a true ULX nature is actually not excluded, at least in cases without double radio-counterpart candidates. In fact, nearly half of these candidates appear compact or extended and are projected against HII regions in the galaxy spiral arms. This could be consistent with physical scenarios where ULX sources are linked to end products of very massive star evolution or binary systems with strong beaming effects. Further investigation will be needed to shed more light on this open question.

Acknowledgements. The authors acknowledge support by the DGI of the Ministerio de Educación y Ciencia (Spain) under grant AYA2004-07171-C0202, FEDER funds, and Plan Andaluz de Investigación of Junta de Andalucía as research group FQM322. This research made use of the SIMBAD database, operated at the CDS, Strasbourg, France. The research of DPR has been supported by the Education Council of Junta de Andalucía (Spain). We also thank Josep M. Paredes (Univ. of Barcelona) for his helpful comments and discussion.

\section{References}

Bignell, R. C., \& Seaquist, E. R. 1983, ApJ, 270, 140

Colbert, E. J. M., \& Ptak, A. F. 2002, ApJS, 143, 25

Corbel, S., Nowak, M. A., Fender, R. P., Tzioumis, A. K., \& Markoff, S. 2003, A\&A, 400, 1007

Fabbiano, G. 1989, ARA\&A, 27, 87

Fabbiano, G. 2004, RMxAC, 20, 46

Fabbiano, G., \& White, N. E. 2006, in Compact Stellar X-Ray Sources. Cambridge (No. 39) [arXiv: astro-ph/0307077]

Falcke, H., Körding, E., \& Markoff, S. 2004, A\&A, 414, 895

Gallo, E., Fender, R. P., \& Pooley, G. G. 2003, MNRAS, 344, 60

Jenkins, L. P., Roberts, T. P., Warwick, R. S., et al. 2004, MNRAS, 349, 404

Kaaret, P., Corbel, S., Prestwich, A. H., \& Zezas, A. 2003, Science, 299, 365

Kaaret, P., Ward, M. J., \& Zezas, A. 2004, MNRAS, 351, L83

Kaaret, P., Simet, M. G., \& Lang, C. C. 2006, Science, 311, 491

Körding, E., Falcke, H., \& Markoff, S. 2002, A\&A, 382, L13

Körding, E., Colbert, E., \& Falcke, H. 2005, A\&A, 436, 427

Lasker, B. M., Strurch, G. R., McLean, B. J., et al. 1990, AJ, 99, 2019

Liu, J.-F., \& Bregman, J. N. 2001, A\&AS, 198, L5601

Liu, J.-F., \& Bregman, J. N. 2005, ApJS, 175, 59

Liu, J.-F., Bregman, J. N., Lloyd-Davies, E., et al. 2005, ApJ, 621, L17

Liu, Q. Z., \& Mirabel, I. F. 2005, A\&A, 429, 1125

Madau, P., \& Rees, M. J. 2001, ApJ, 551, L27

Merloni, A., Heinz, S., \& Di Matteo, T. 2003, MNRAS, 345, 1057

Miller, M. C., \& Colbert, E. J. M. 2004, Int. J. Mod. Phys. D, 13, 1

Miller, N. A., Mushotzky, R. F., \& Neff, S. G. 2005, ApJ, 623, L109

Miyoshi, M., Moran, J., Herrnstein, J., et al. 1995, Nature, 373, 127

Mushotzky, R. 2004, Prog. Theor. Phys. Supp., 155, 27

Pakull, M. W., \& Mirioni, L. 2003, RMAA, 15, 197

Soria, R., Cropper, M., \& Motch, C. 2005, ChJAA, Vol. 5 Suppl., 153

Summers, L. K., Stevens, I. R., Strickland, D. K., \& Heckman, T. M. 2003, MNRAS, 342, 690

White, R. L., Becker, R. H., Helfand, D. J., \& Gregg, M. D. 1997, ApJ, 475, 479 


\section{Online Material}


J. R. Sánchez-Sutil et al.: A catalogue of ULX coincidences with FIRST radio sources (RN), Online Material p 2

Table 3. Candidate radio counterparts of ULX sources found after the cross-identification process.

\begin{tabular}{|c|c|c|c|c|c|c|c|c|c|}
\hline NGC & $\begin{array}{l}\text { ULX } \\
\text { Name }\end{array}$ & $\begin{array}{c}\alpha_{\mathrm{J} 2000.0} \\
(\text { FIRST) }\end{array}$ & $\begin{array}{c}\delta_{\mathrm{J} 2000.0} \\
\text { (FIRST) }\end{array}$ & $\begin{array}{c}\text { Radio vs. } \\
\text { X-ray } \\
\text { offset }\end{array}$ & $\begin{array}{c}\text { Peak } \\
\text { Flux Density } \\
\text { (mJy) }\end{array}$ & $\begin{array}{l}\text { Integrated } \\
\text { Flux Density } \\
(\mathrm{mJy})\end{array}$ & $\begin{array}{c}\text { X-ray } \\
\text { Luminosity } \\
\left(10^{39} \mathrm{erg} \mathrm{s}^{-1}\right)\end{array}$ & $\begin{array}{c}\text { Procedence } \\
\text { catalog(s) }\end{array}$ & Comments $^{1}$ \\
\hline 1052 & $\mathrm{X} 1$ & $02^{\mathrm{h}} 41^{\mathrm{m}} 04^{\mathrm{s}} .81$ & $-08^{\circ} 15^{\prime} 20^{\prime \prime} 9$ & 2 .' $65^{\prime}$ & 967.08 & 1017.22 & 32.8 & LB & $\mathrm{N}$ \\
\hline 1068 & $\mathrm{X} 1$ & $02^{\mathrm{h}} 42^{\mathrm{m}} 40^{\mathrm{s}} \cdot 65$ & $-00^{\circ} 00^{\prime} 49^{\prime \prime} .2$ & 5. 25 & 797.54 & 1917.93 & 1330 & LB, LM & $\mathrm{N}$ \\
\hline 1073 & $\mathrm{X} 1$ & $02^{\mathrm{h}} 43^{\mathrm{m}} 39^{\mathrm{s}} .60$ & $+01^{\circ} 21^{\prime} 09^{\prime \prime} 5$ & 2.92 & 39.25 & 55.98 & 3.16 & LB & QSO \\
\hline 2782 & $\mathrm{X} 1$ & $09^{\mathrm{h}} 14^{\mathrm{m}} 05^{\mathrm{s}} .08$ & $+40^{\circ} 06^{\prime} 49^{\prime \prime} 4$ & $2 . " 18$ & 34.48 & 107.44 & 98.5 & LB & $\mathrm{N}$ \\
\hline 2903 & $\mathrm{X} 1$ & $09^{\mathrm{h}} 32^{\mathrm{m}} 10^{\mathrm{s}} .09$ & $+21^{\circ} 30^{\prime} 04^{\prime \prime} 3$ & $2 . \prime 24$ & 19.03 & 63.39 & 5.26 & LB & $\mathrm{N}$ \\
\hline 2974 & $\mathrm{X} 1$ & $09^{\mathrm{h}} 42^{\mathrm{m}} 33^{\mathrm{s}} .28$ & $-03^{\circ} 41^{\prime} 56^{\prime \prime} 9$ & $0 . \prime 24$ & 5.88 & 7.54 & 31.7 & LB & $\mathrm{N}$ \\
\hline 3190 & $\mathrm{X} 1$ & $10^{\mathrm{h}} 18^{\mathrm{m}} 05^{\mathrm{s}} .63$ & $+21^{\circ} 49^{\prime} 55^{\prime \prime} 1$ & 6." 65 & 2.50 & 4.03 & 8.79 & LB & $\mathrm{N}$ \\
\hline 3226 & $\mathrm{X} 1$ & $10^{\mathrm{h}} 23^{\mathrm{m}} 27^{\mathrm{s}} .01$ & $+19^{\circ} 53^{\prime} 54^{\prime \prime} 8$ & 1."96 & 4.45 & 4.49 & 18 & LB & $\mathrm{N}$ \\
\hline 3227 & $\mathrm{X} 1$ & $10^{\mathrm{h}} 23^{\mathrm{m}} 30^{\mathrm{s}} .58$ & $+19^{\circ} 51^{\prime} 54^{\prime \prime} 5$ & 1." 12 & 74.39 & 82.85 & 489 & LB & $\mathrm{N}$ \\
\hline 3310 & $\mathrm{X} 1$ & $10^{\mathrm{h}} 38^{\mathrm{m}} 45^{\mathrm{s}} .97$ & $+53^{\circ} 30^{\prime} 14^{\prime \prime} 3$ & 4." 38 & 7.68 & 102.87 & 83.1 & LB, LM & $\mathrm{N}$ \\
\hline 3395 & $\mathrm{X} 1$ & $10^{\mathrm{h}} 49^{\mathrm{m}} 50^{\mathrm{s}} .14$ & $+32^{\circ} 59^{\prime} 00^{\prime \prime} 1$ & 7.' 18 & 1.28 & 20.64 & 5.62 & LM & $\mathrm{N}$ \\
\hline 3627 & $\mathrm{X} 1$ & $11^{\mathrm{h}} 20^{\mathrm{m}} 15^{\mathrm{s}} .00$ & $+12^{\circ} 59^{\prime} 29^{\prime \prime} 6$ & 8." 31 & 12.01 & 13.40 & 2.21 & LB & $\mathrm{N}$ \\
\hline 3665 & $\mathrm{X} 1$ & $11^{\mathrm{h}} 24^{\mathrm{m}} 43^{\mathrm{s}} .03$ & $+38^{\circ} 45^{\prime} 51_{\prime \prime \prime} 9$ & 9." 87 & 12.47 & 24.10 & 33.5 & LB & $\mathrm{N}$ \\
\hline 3690 & $\mathrm{X} 2$ & $11^{\mathrm{h}} 28^{\mathrm{m}} 30^{\mathrm{s}} \cdot 95$ & $+58^{\circ} 33^{\prime} 40^{\prime \prime} 8$ & 4." 16 & 56.22 & 83.49 & 6.6 & LM & $\mathrm{N}^{2}$ \\
\hline 3690 & $\mathrm{X} 3$ & $11^{\mathrm{h}} 28^{\mathrm{m}} 30^{\mathrm{s}} .95$ & $+58^{\circ} 33^{\prime} 40^{\prime \prime} 8$ & $4 . " 20$ & 56.22 & 83.49 & 23.9 & LM & $\mathrm{N}^{2}$ \\
\hline 3690 & $\mathrm{X} 8$ & $11^{\mathrm{h}} 28^{\mathrm{m}} 33^{\mathrm{s}} .64$ & $+58^{\circ} 33^{\prime} 46^{\prime \prime} 2$ & 4." 13 & 169.31 & 282.27 & 3.5 & LM & $\mathrm{N}^{2}$ \\
\hline 3690 & $\mathrm{X} 10$ & $11^{\mathrm{h}} 28^{\mathrm{m}} 33^{\mathrm{s}} .64$ & $+58^{\circ} 33^{\prime} 46^{\prime \prime} 2$ & $2 . \prime 04$ & 169.31 & 282.27 & 5.8 & LM & $\mathrm{N}^{2}$ \\
\hline 3690 & $\mathrm{X} 11$ & $11^{\mathrm{h}} 28^{\mathrm{m}} 33^{\mathrm{s}} .64$ & $+58^{\circ} 33^{\prime} 46^{\prime \prime} 2$ & 5."07 & 169.31 & 282.27 & 4.1 & LM & $\mathrm{N}^{2}$ \\
\hline 3690 & $\mathrm{X} 12$ & $11^{\mathrm{h}} 28^{\mathrm{m}} 33^{\mathrm{s}} \cdot 64$ & $+58^{\circ} 33^{\prime} 46^{\prime \prime} 2$ & 7." 11 & 169.31 & 282.27 & 2.9 & LM & $\mathrm{N}^{2}$ \\
\hline 3738 & $\mathrm{X} 1$ & $11^{\mathrm{h}} 35^{\mathrm{m}} 45^{\mathrm{s}} \cdot 42$ & $+54^{\circ} 33^{\prime} 15^{\prime \prime} 4$ & $0 . \prime 06$ & 61.37 & 94.11 & 0.371 & LB & QSO? \\
\hline 3982 & $\mathrm{X} 1$ & $11^{\mathrm{h}} 56^{\mathrm{m}} 28^{\mathrm{s}} .15$ & $+55^{\circ} 07^{\prime} 31^{\prime \prime} 0$ & $1 . " 34$ & 3.07 & 3.97 & 5.13 & LB & $\mathrm{N}$ \\
\hline 3998 & $\mathrm{X} 1$ & $11^{\mathrm{h}} 57^{\mathrm{m}} 56^{\mathrm{s}} .13$ & $+55^{\circ} 27^{\prime} 13^{\prime \prime} 0$ & $1 . " 85$ & 93.45 & 98.51 & 259 & LB & $\mathrm{N}$ \\
\hline 4051 & $\mathrm{X} 1$ & $12^{\mathrm{h}} 03^{\mathrm{m}} 09^{\mathrm{s}} .59$ & $+44^{\circ} 31^{\prime} 52^{\prime \prime} .5$ & $2 . " 29$ & 12.30 & 19.34 & 2770 & LB & $\mathrm{N}$ \\
\hline 4088 & $\mathrm{X} 1$ & $12^{\mathrm{h}} 05^{\mathrm{m}} 31^{\mathrm{s}} .70$ & $+50^{\circ} 32^{\prime} 46^{\prime \prime} 8$ & 3." 62 & 1.87 & 5.07 & 5.86 & $\mathrm{LB}, \mathrm{LM}, \mathrm{CP}$ & HII? \\
\hline 4151 & $\mathrm{X} 1$ & $12^{\mathrm{h}} 10^{\mathrm{m}} 32^{\mathrm{s}} .55$ & $+39^{\circ} 24^{\prime} 21^{\prime \prime} 0$ & $0 . \prime 24$ & 290.78 & 331.29 & 1330 & LB & $\mathrm{N}$ \\
\hline 4156 & $\mathrm{X} 1$ & $12^{\mathrm{h}} 10^{\mathrm{m}} 49^{\mathrm{s}} \cdot 61$ & $+39^{\circ} 28^{\prime} 22^{\prime \prime} 2$ & $1 . \prime 28$ & 2.46 & 2.93 & 4.32 & LB & $\mathrm{N}$ \\
\hline 4203 & $\mathrm{X} 1$ & $12^{\mathrm{h}} 15^{\mathrm{m}} 05^{\mathrm{s}} .06$ & $+33^{\circ} 11^{\prime} 50^{\prime \prime} 7$ & $1 . ' 25$ & 6.94 & 7.72 & 109 & LB & $\mathrm{N}$ \\
\hline 4235 & $\mathrm{X} 1$ & $12^{\mathrm{h}} 17^{\mathrm{m}} 09^{\mathrm{s}} .86$ & $+07^{\circ} 11^{\prime} 29^{\prime \prime} 7$ & 3." 00 & 4.66 & 5.10 & 1270 & LB & $\mathrm{N}$ \\
\hline 4258 & X6 & $12^{\mathrm{h}} 18^{\mathrm{m}} 46^{\mathrm{s}} .32$ & $+47^{\circ} 14^{\prime} 20^{\prime \prime} 4$ & 3." 90 & 7.14 & 12.44 & 0.572 & LB & QSO? \\
\hline 4261 & $\mathrm{X} 1$ & $12^{\mathrm{h}} 19^{\mathrm{m}} 23^{\mathrm{s}} \cdot 23$ & $+05^{\circ} 49^{\prime} 29^{\prime \prime} 9$ & 3." 99 & 135.57 & 140.46 & 229 & LB & $\mathrm{N}$ \\
\hline 4278 & $\mathrm{X} 1$ & $12^{\mathrm{h}} 20^{\mathrm{m}} 06^{\mathrm{s}} .82$ & $+29^{\circ} 16^{\prime} 50^{\prime \prime} 7$ & $1 . \prime 97$ & 389.38 & 402.00 & 42.8 & LB & $\mathrm{N}$ \\
\hline 4303 & $\mathrm{X} 1$ & $12^{\mathrm{h}} 21^{\mathrm{m}} 54^{\mathrm{s}} .91$ & $+04^{\circ} 28^{\prime} 26^{\prime \prime} 7$ & 1." 80 & 4.85 & 9.74 & 8.51 & LB & $\mathrm{N}$ \\
\hline 4321 & $\mathrm{X} 1$ & $12^{\mathrm{h}} 22^{\mathrm{m}} 54^{\mathrm{s}} .95$ & $+15^{\circ} 49^{\prime} 20^{\prime \prime} 7$ & 5." 17 & 3.40 & 37.35 & 17.2 & LB, LM & $\mathrm{N}$ \\
\hline 4321 & $\mathrm{X} 2$ & $12^{\mathrm{h}} 22^{\mathrm{m}} 58^{\mathrm{s}} .66$ & $+15^{\circ} 47^{\prime} 51^{\prime \prime} 8$ & $2 . " 50$ & 5.18 & 5.64 & 1.57 & LB & SNR \\
\hline 4374 & $\mathrm{X} 1$ & $12^{\mathrm{h}} 25^{\mathrm{m}} 03^{\mathrm{s}} .72$ & $+12^{\circ} 53^{\prime} 21^{\prime \prime} .8$ & 7."77 & 105.14 & 196.92 & 137 & LB & $\mathrm{N}$ \\
\hline 4388 & $\mathrm{X} 1$ & $12^{\mathrm{h}} 25^{\mathrm{m}} 46^{\mathrm{s}} .74$ & $+12^{\circ} 39^{\prime} 42^{\prime \prime} 7$ & 7!'56 & 24.35 & 35.84 & 23 & LB & $\mathrm{N}$ \\
\hline 4395 & $\mathrm{X} 2$ & $12^{\mathrm{h}} 25^{\mathrm{m}} 32^{\mathrm{s}} .31$ & $+33^{\circ} 25^{\prime} 34^{\prime \prime} 0$ & $0 . \prime 05$ & 1.35 & 1.28 & 0.308 & LB & - \\
\hline 4435 & $\mathrm{X} 1$ & $12^{\mathrm{h}} 27^{\mathrm{m}} 40^{\mathrm{s}} .54$ & $+13^{\circ} 04^{\prime} 44^{\prime \prime} 9$ & 3." 45 & 1.73 & 2.16 & 6.67 & LB & $\mathrm{N}$ \\
\hline 4438 & $\mathrm{X} 1$ & $12^{\mathrm{h}} 27^{\mathrm{m}} 45^{\mathrm{s}} .57$ & $+13^{\circ} 00^{\prime} 32^{\prime \prime} 9$ & $2 . " 43$ & 56.18 & 76.24 & 25.7 & LB & $\mathrm{N}$ \\
\hline 4449 & $\mathrm{X} 4$ & $12^{\mathrm{h}} 28^{\mathrm{m}} 10^{\mathrm{s}} .96$ & $+44^{\circ} 06^{\prime} 48^{\prime \prime} 4$ & 3." 30 & 9.27 & 9.81 & 0.45 & LB & S? \\
\hline 4472 & $\mathrm{X} 1$ & $12^{\mathrm{h}} 29^{\mathrm{m}} 46^{\mathrm{s}} .74$ & $+08^{\circ} 00^{\prime} 01^{\prime \prime} 6$ & $5 . \prime 25$ & 98.39 & 123.70 & 181 & LB & $\mathrm{N}$ \\
\hline 4485 & X3 & $12^{\mathrm{h}} 30^{\mathrm{m}} 31^{\mathrm{s}} .40$ & $+41^{\circ} 39^{\prime} 08^{\prime \prime} 6$ & 7.' 16 & 1.29 & 12.88 & 4.6 & LM & $\mathrm{E}$ \\
\hline 4526 & $\mathrm{X} 1$ & $12^{\mathrm{h}} 34^{\mathrm{m}} 02^{\mathrm{s}} .93$ & $+07^{\circ} 41^{\prime} 58^{\prime \prime} 6$ & 7!' 13 & 3.76 & 12.00 & 7.16 & LB & $\mathrm{N}$ \\
\hline 4536 & $\mathrm{X} 1$ & $12^{\mathrm{h}} 34^{\mathrm{m}} 27^{\mathrm{s}} .07$ & $+02^{\circ} 11^{\prime} 18^{\prime \prime} 0$ & $2 . " 44$ & 44.80 & 131.82 & 5.76 & LB & $\mathrm{N}$ \\
\hline 4552 & $\mathrm{X} 1$ & $12^{\mathrm{h}} 35^{\mathrm{m}} 39^{\mathrm{s}} .80$ & $+12^{\circ} 33^{\prime} 22^{\prime \prime} 9$ & 3." 09 & 107.95 & 112.78 & 82.6 & LB & $\mathrm{N}$ \\
\hline 4559 & $\mathrm{X} 4$ & $12^{\mathrm{h}} 35^{\mathrm{m}} 56^{\mathrm{s}} .30$ & $+27^{\circ} 59^{\prime} 26^{\prime \prime} 4$ & 5.35 & 1.12 & 0.90 & 0.207 & LB & HII? \\
\hline 4565 & $\mathrm{X} 1$ & $12^{\mathrm{h}} 36^{\mathrm{m}} 20^{\mathrm{s}} .77$ & $+25^{\circ} 59^{\prime} 15^{\prime \prime} 8$ & $1 . " 60$ & 1.48 & 1.88 & 12.1 & LB & $\mathrm{N}$ \\
\hline 4569 & $\mathrm{X} 1$ & $12^{\mathrm{h}} 36^{\mathrm{m}} 49^{\mathrm{s}} .82$ & $+13^{\circ} 09^{\prime} 46^{\prime \prime} 7$ & $2 . " 13$ & 7.69 & 14.36 & 13.3 & LB & $\mathrm{N}$ \\
\hline 4593 & $\mathrm{X} 1$ & $12^{\mathrm{h}} 39^{\mathrm{m}} 39^{\mathrm{s}} \cdot 44$ & $-05^{\circ} 20^{\prime} 39^{\prime \prime} .2$ & 4." 51 & 2.82 & 5.20 & 15600 & LB & $\mathrm{N}$ \\
\hline 4631 & $\mathrm{X} 2$ & $12^{\mathrm{h}} 42^{\mathrm{m}} 10^{\mathrm{s}} .59$ & $+32^{\circ} 32^{\prime} 37^{\prime \prime} .^{\prime} 0$ & 6." 57 & 3.93 & 38.34 & 1.34 & LM & $\mathrm{N}^{3}$ \\
\hline 4636 & $\mathrm{X} 1$ & $12^{\mathrm{h}} 42^{\mathrm{m}} 49^{\mathrm{s}} .96$ & $+02^{\circ} 41^{\prime} 16^{\prime \prime} 2$ & 4." 66 & 14.03 & 56.91 & 148 & $\mathrm{LB}$ & $\mathrm{N}$ \\
\hline 4649 & $\mathrm{X} 1$ & $12^{\mathrm{h}} 43^{\mathrm{m}} 39^{\mathrm{s}} .98$ & $+11^{\circ} 33^{\prime} 09^{\prime \prime} 6$ & 3." 59 & 15.26 & 16.54 & 229 & LB & $\mathrm{N}$ \\
\hline 4736 & $\mathrm{X} 1$ & $12^{\mathrm{h}} 50^{\mathrm{m}} 53^{\mathrm{s}} .06$ & $+41^{\circ} 07^{\prime} 13 .^{\prime \prime} 6$ & 3." 58 & 8.91 & 13.51 & 13.3 & LB & $\mathrm{N}$ \\
\hline 4826 & $\mathrm{X} 1$ & $12^{\mathrm{h}} 56^{\mathrm{m}} 43^{\mathrm{s}} \cdot 62$ & $+21^{\circ} 41^{\prime} 00^{\prime \prime} 3$ & 4." 13 & 7.95 & 36.06 & 4.49 & LB & $\mathrm{N}$ \\
\hline 4861 & $\mathrm{X} 2$ & $12^{\mathrm{h}} 59^{\mathrm{m}} 00^{\mathrm{s}} \cdot 35$ & $+34^{\circ} 50^{\prime} 42^{\prime \prime} 9$ & 1." 97 & 3.88 & 6.01 & 8.4 & LB, LM, CP & HII \\
\hline 5005 & $\mathrm{X} 1$ & $13^{\mathrm{h}} 10^{\mathrm{m}} 56^{\mathrm{s}} \cdot 23$ & $+37^{\circ} 03^{\prime} 33^{\prime \prime} 1$ & 7.'97 & 35.90 & 45.81 & 58.3 & LB & $\mathrm{N}$ \\
\hline 5033 & $\mathrm{X} 1$ & $13^{\mathrm{h}} 13^{\mathrm{m}} 27^{\mathrm{s}} .50$ & $+36^{\circ} 35^{\prime} 38^{\prime \prime} 1$ & 1."79 & 8.74 & 23.59 & 135 & LB & $\mathrm{N}$ \\
\hline 5055 & $\mathrm{X} 1$ & $13^{\mathrm{h}} 15^{\mathrm{m}} 49^{\mathrm{s}} \cdot 21$ & $+42^{\circ} 01^{\prime} 46^{\prime \prime} 3$ & $1 . " 31$ & 1.81 & 20.23 & 2.2 & LB & $\mathrm{N}$ \\
\hline 5194 & $\mathrm{X} 1$ & $13^{\mathrm{h}} 29^{\mathrm{m}} 52^{\mathrm{s}} .02$ & $+47^{\circ} 11^{\prime} 43^{\prime \prime} 5$ & 6." 62 & 1.26 & 16.54 & 17.2 & LB & $\mathrm{N}$ \\
\hline 5194 & $\mathrm{X} 3$ & $13^{\mathrm{h}} 29^{\mathrm{m}} 50^{\mathrm{s}} \cdot 65$ & $+47^{\circ} 11^{\prime} 54^{\prime \prime} 6$ & 0.51 & 1.20 & 3.20 & 3.95 & LM & $\mathrm{N}^{3}$ \\
\hline 5195 & $\mathrm{X} 1$ & $13^{\mathrm{h}} 29^{\mathrm{m}} 59^{\mathrm{s}} .52$ & $+47^{\circ} 15^{\prime} 58^{\prime \prime} 5$ & 4." 33 & 5.87 & 9.17 & 3.27 & LB & $\mathrm{N}$ \\
\hline
\end{tabular}


J. R. Sánchez-Sutil et al.: A catalogue of ULX coincidences with FIRST radio sources (RN), Online Material p 3

Table 3. continued.

\begin{tabular}{|c|c|c|c|c|c|c|c|c|c|}
\hline NGC & $\begin{array}{l}\text { ULX } \\
\text { Name }\end{array}$ & $\begin{array}{c}\alpha_{\mathrm{J} 2000.0} \\
\text { (FIRST) }\end{array}$ & $\begin{array}{c}\delta_{\mathrm{J} 2000.0} \\
(\text { FIRST) }\end{array}$ & $\begin{array}{c}\text { Radio vs. } \\
\text { X-ray } \\
\text { offset }\end{array}$ & $\begin{array}{c}\text { Peak } \\
\text { Flux Density } \\
(\mathrm{mJy})\end{array}$ & $\begin{array}{c}\text { Integrated } \\
\text { Flux Density } \\
(\mathrm{mJy})\end{array}$ & $\begin{array}{c}\text { X-ray } \\
\text { Luminosity } \\
\left(10^{39} \mathrm{erg} \mathrm{s}^{-1}\right)\end{array}$ & $\begin{array}{c}\text { Procedence } \\
\text { catalog(s) }\end{array}$ & $\overline{\text { Comments }}^{1}$ \\
\hline 5273 & $\mathrm{X} 1$ & $13^{\mathrm{h}} 42^{\mathrm{m}} 08^{\mathrm{s}} .35$ & $+35^{\circ} 39^{\prime} 15^{\prime \prime} 4$ & 2.29 & 2.60 & 2.90 & 4.72 & $\overline{L B}$ & $\mathrm{~N}$ \\
\hline 5322 & $\mathrm{X} 1$ & $13^{\mathrm{h}} 49^{\mathrm{m}} 15^{\mathrm{s}} .12$ & $+60^{\circ} 11^{\prime} 32^{\prime \prime}{ }^{\prime} 8$ & 8." 82 & 7.19 & 8.78 & 29.7 & LB & $\mathrm{N}$ \\
\hline 5350 & $\mathrm{X} 1$ & $13^{\mathrm{h}} 53^{\mathrm{m}} 21^{\mathrm{s}} \cdot 60$ & $+40^{\circ} 21^{\prime} 50^{\prime \prime} 5$ & 4." 27 & 2.85 & 4.03 & 21.4 & LB & $\mathrm{N}$ \\
\hline 5353 & $\mathrm{X} 1$ & $13^{\mathrm{h}} 53^{\mathrm{m}} 26^{\mathrm{s}} \cdot 69$ & $+40^{\circ} 16^{\prime} 58^{\prime \prime} 9$ & $6 . " 04$ & 36.19 & 38.30 & 106 & LB & $\mathrm{N}$ \\
\hline 5457 & X9 & $14^{\mathrm{h}} 03^{\mathrm{m}} 41^{\mathrm{s}} .42$ & $+54^{\circ} 19^{\prime} 05^{\prime \prime} 2$ & 3." 40 & 6.63 & 11.45 & 0.307 & LB, LM & HII? \\
\hline 5457 & $\mathrm{X} 17$ & $14^{\mathrm{h}} 02^{\mathrm{m}} 28^{\mathrm{s}} .12$ & $+54^{\circ} 16^{\prime} 27^{\prime \prime} 3$ & $4 . " 30$ & 1.10 & 1.97 & 0.891 & LB, LM & HII? \\
\hline 5457 & $\mathrm{X} 26$ & $14^{\mathrm{h}} 04^{\mathrm{m}} 29^{\mathrm{s}} .14$ & $+54^{\circ} 23^{\prime} 53^{\prime \prime} .^{\prime} 4$ & 4." 07 & 2.65 & 4.05 & 0.615 & $\mathrm{LB}, \mathrm{LM}$ & HNR? SSS? \\
\hline 5457 & X29 & $14^{\mathrm{h}} 04^{\mathrm{m}} 00^{\mathrm{s}} .78$ & $+54^{\circ} 09^{\prime} 11^{\prime \prime} 3$ & 6." 36 & 3.68 & 5.43 & 0.228 & LB & FRII? \\
\hline 5506 & $\mathrm{X} 1$ & $14^{\mathrm{h}} 13^{\mathrm{m}} 14^{\mathrm{s}} .88$ & $-03^{\circ} 12^{\prime} 27^{\prime \prime} 5$ & $1 . " 22$ & 309.97 & 331.36 & 455 & LB & $\mathrm{N}$ \\
\hline
\end{tabular}

(1) N: nuclear; QSO: quasar; HII: HII region; SNR: supernova remnant; E: non nuclear but extended; HNR: hypernova remnant; SSS: supersoft source; FRII: Fanaroff-Riley type II.

(2) Multiple coincidences with a single FIRST source.

(3) Very close to nucleus, but not exactly coincident. 
J. R. Sánchez-Sutil et al.: A catalogue of ULX coincidences with FIRST radio sources (RN), Online Material p 4

Table 4. Adopted distances to the galaxies listed in Table 2.

\begin{tabular}{ccc}
\hline \hline NGC & Redshift $\left(\times 10^{-4}\right)$ & Distance $(\mathrm{Mpc})$ \\
\hline 3738 & 7.64 & 3.05 \\
4088 & 25.24 & 10.09 \\
4258 & 14.94 & 5.97 \\
4395 & 10.64 & 4.25 \\
4449 & 6.9 & 2.76 \\
4559 & 27.22 & 10.88 \\
4861 & 27.79 & 11.11 \\
5457 & 8.04 & 3.21 \\
\hline
\end{tabular}


J. R. Sánchez-Sutil et al.: A catalogue of ULX coincidences with FIRST radio sources (RN), Online Material p 5

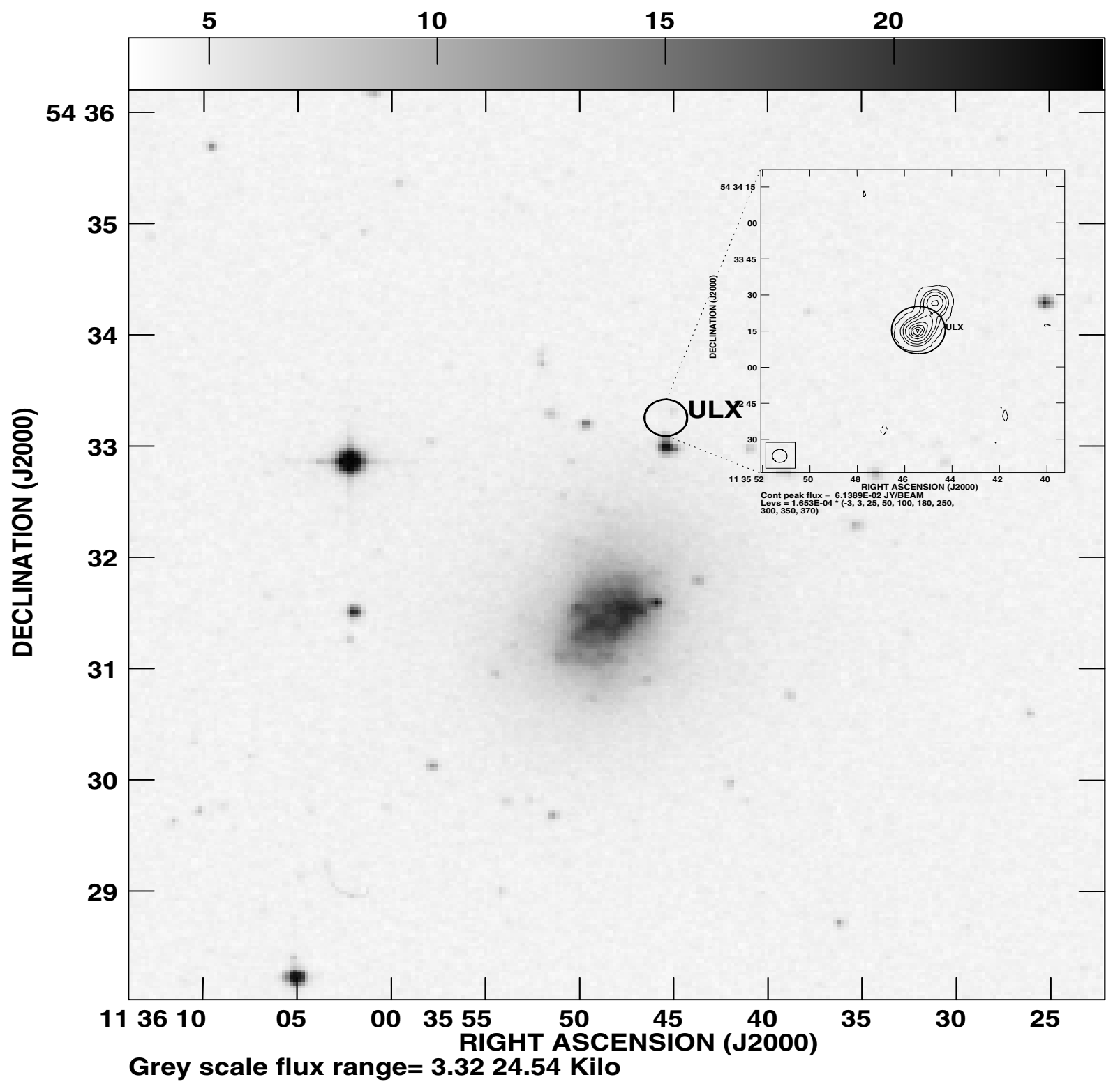

Fig. 1. Radio counterpart candidate of X1 in the field of NGC 3738. The radio image in the inside panel has been contoured in units of the FIRST rms noise, typically $0.15 \mathrm{mJy}_{\text {beam }}^{-1}$. The circle in the panel's lower left corner corresponds to the size of the synthesized beam, about $5^{\prime \prime}$. These values also apply to the rest of the figures. 
J. R. Sánchez-Sutil et al.: A catalogue of ULX coincidences with FIRST radio sources $(R N)$, Online Material p 6

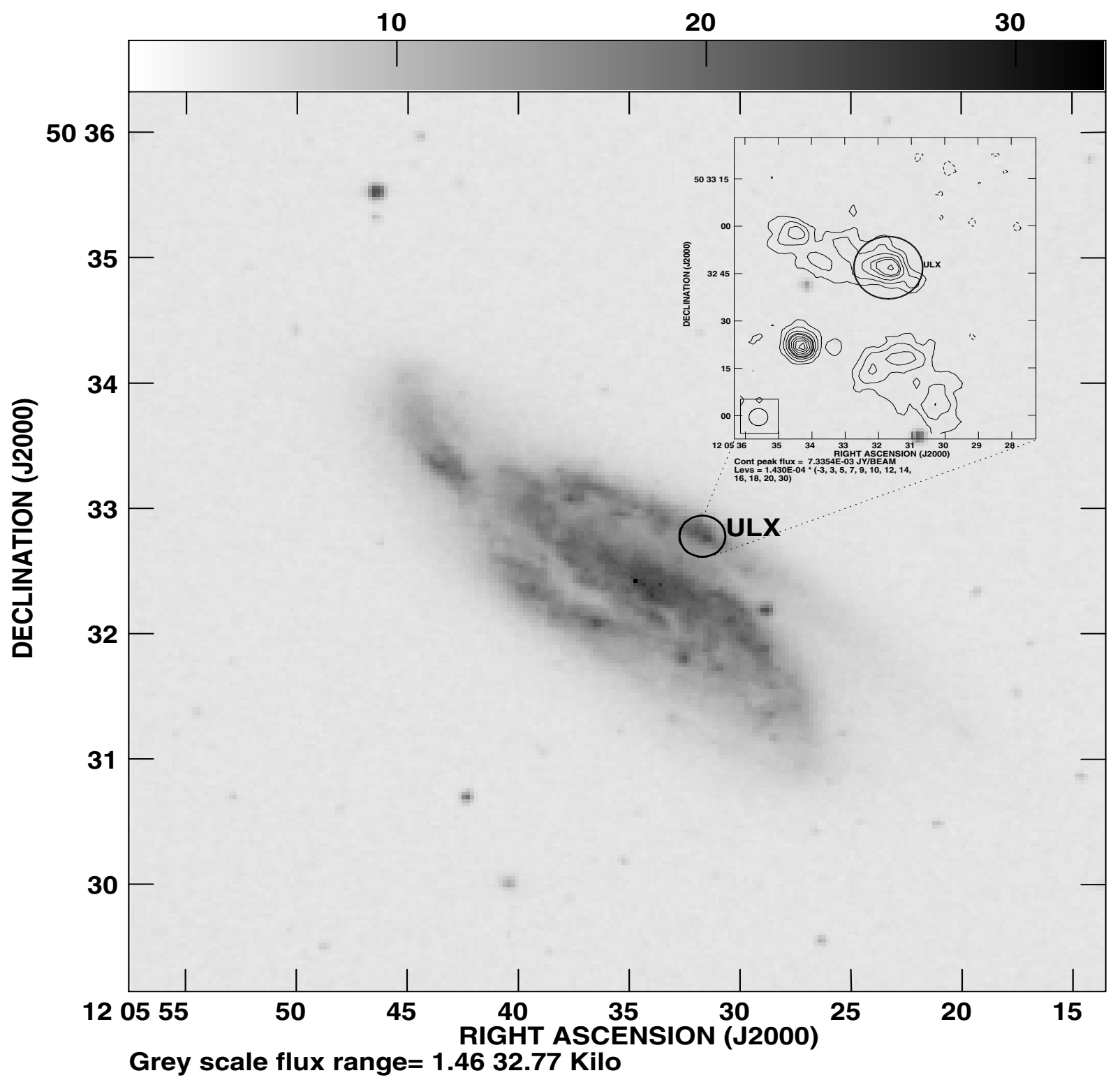

Fig. 2. Coincidence of X1 in the field of NGC 4088 with a conspicuous peak of radio emission in the galaxy spiral arm. 
J. R. Sánchez-Sutil et al.: A catalogue of ULX coincidences with FIRST radio sources (RN), Online Material p 7

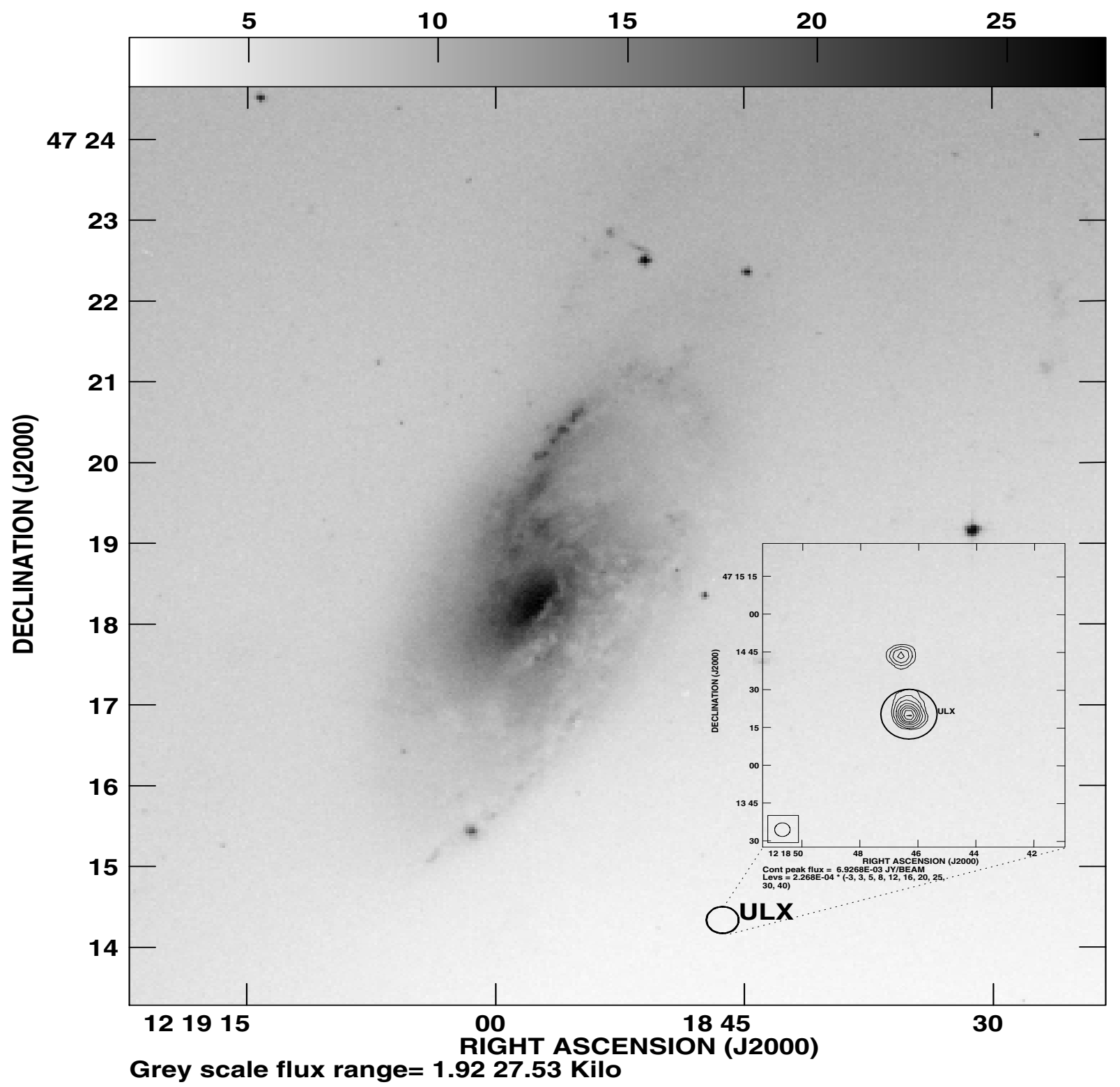

Fig. 3. Radio counterpart candidate of the X6 source in the field of NGC 4258. 
J. R. Sánchez-Sutil et al.: A catalogue of ULX coincidences with FIRST radio sources $(R N)$, Online Material p 8

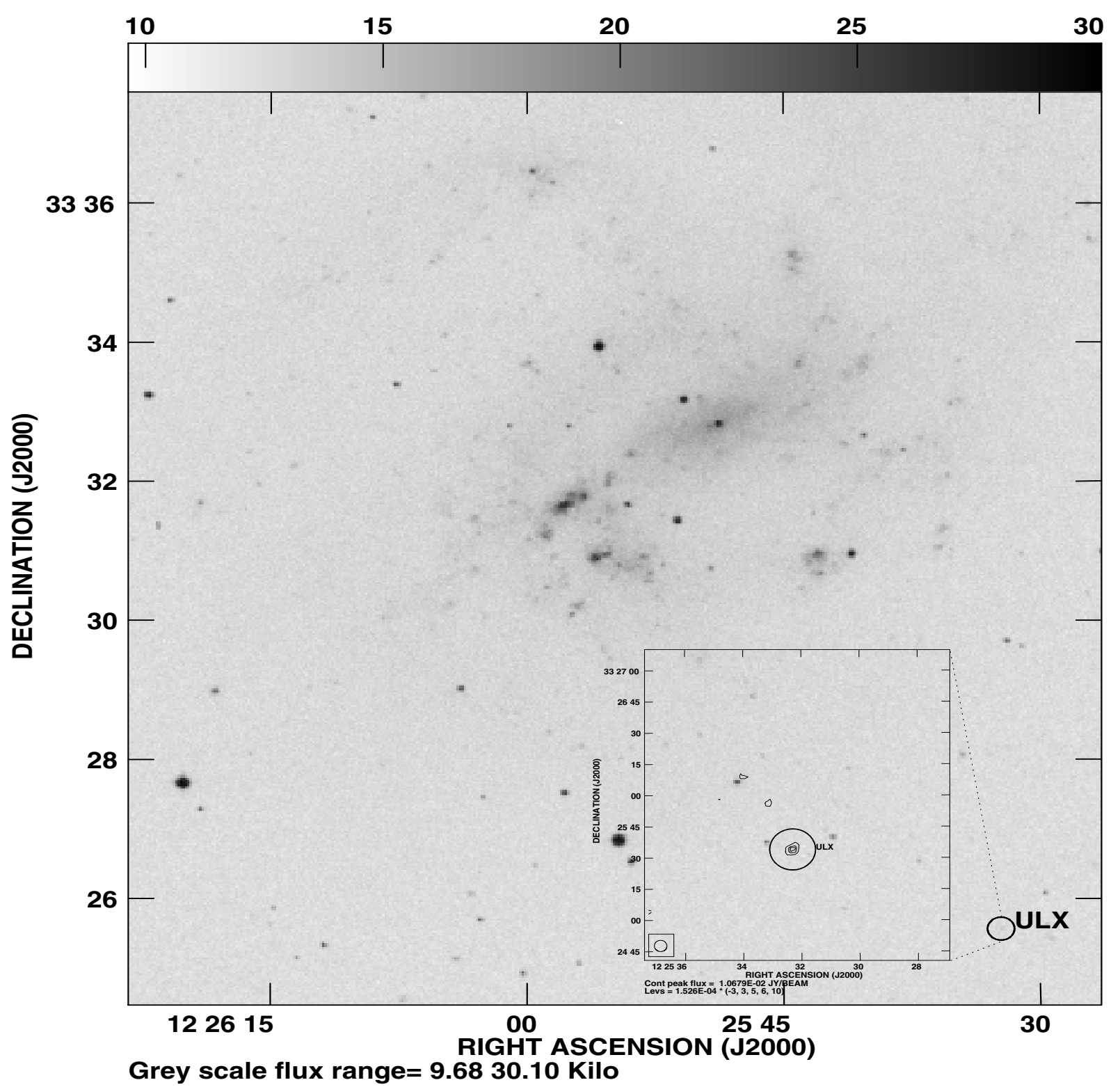

Fig. 4. Radio counterpart candidate of the X2 source in the field of NGC 4395. 
J. R. Sánchez-Sutil et al.: A catalogue of ULX coincidences with FIRST radio sources (RN), Online Material $p 9$

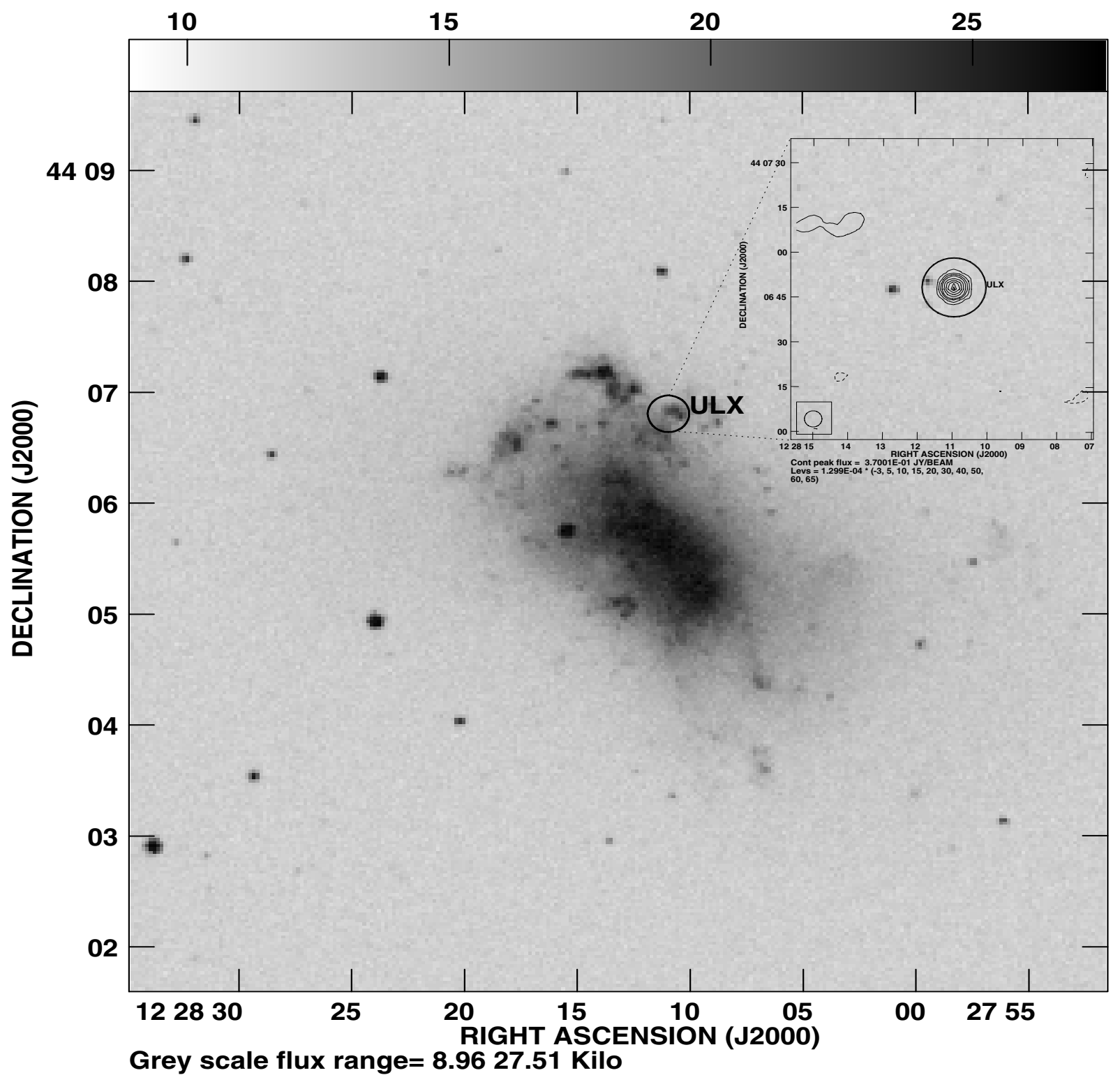

Fig. 5. Radio counterpart candidate of the X4 source in the field of NGC 4449. 
J. R. Sánchez-Sutil et al.: A catalogue of ULX coincidences with FIRST radio sources (RN), Online Material p 10

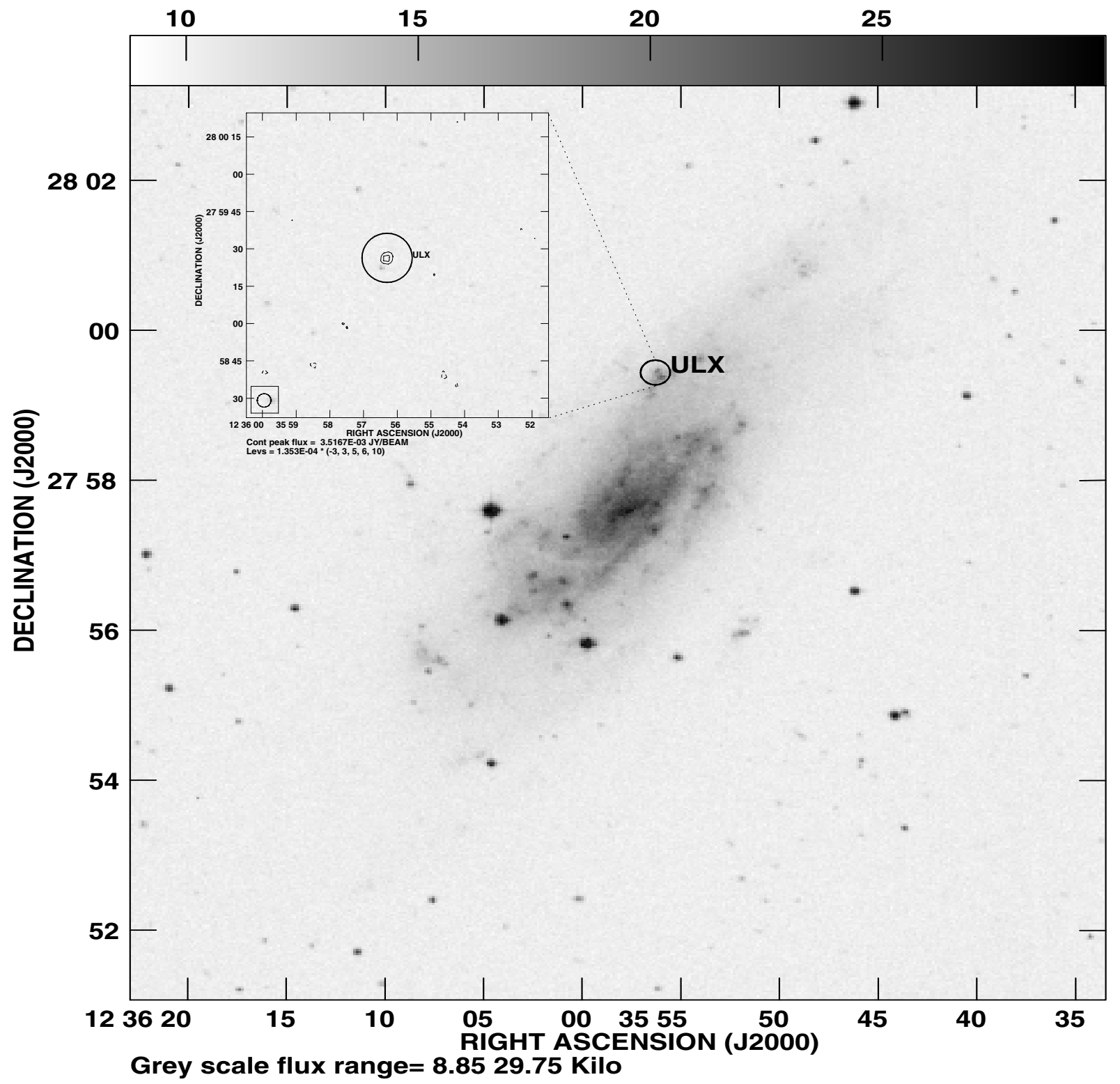

Fig. 6. Radio counterpart candidate of the X4 source in the field of NGC 4559. 
J. R. Sánchez-Sutil et al.: A catalogue of ULX coincidences with FIRST radio sources (RN), Online Material p 11

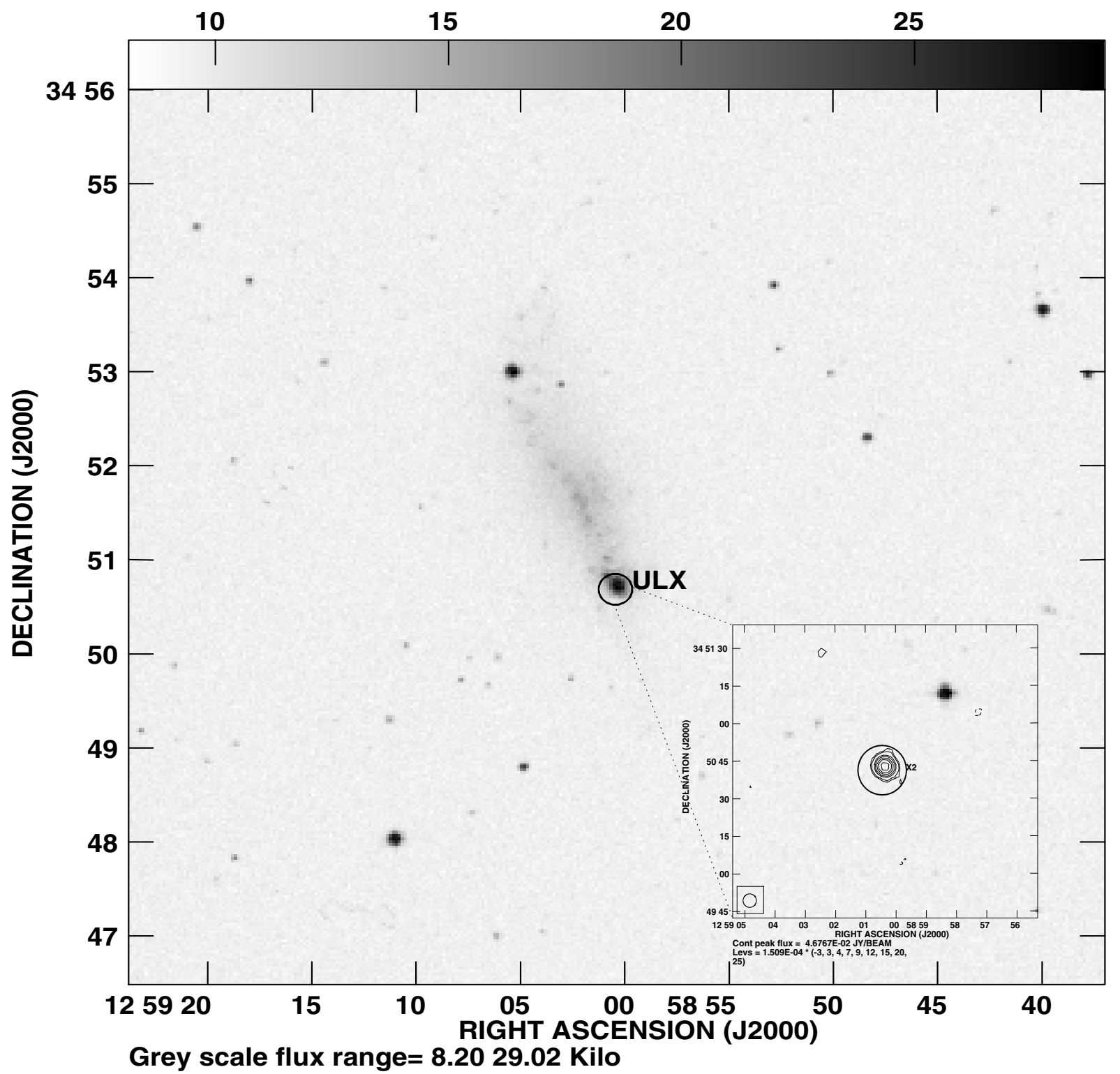

Fig. 7. Radio counterpart candidate of the $\mathrm{X} 2$ source in NGC 4861 in coincidence with a huge HII region. 
J. R. Sánchez-Sutil et al.: A catalogue of ULX coincidences with FIRST radio sources (RN), Online Material p 12

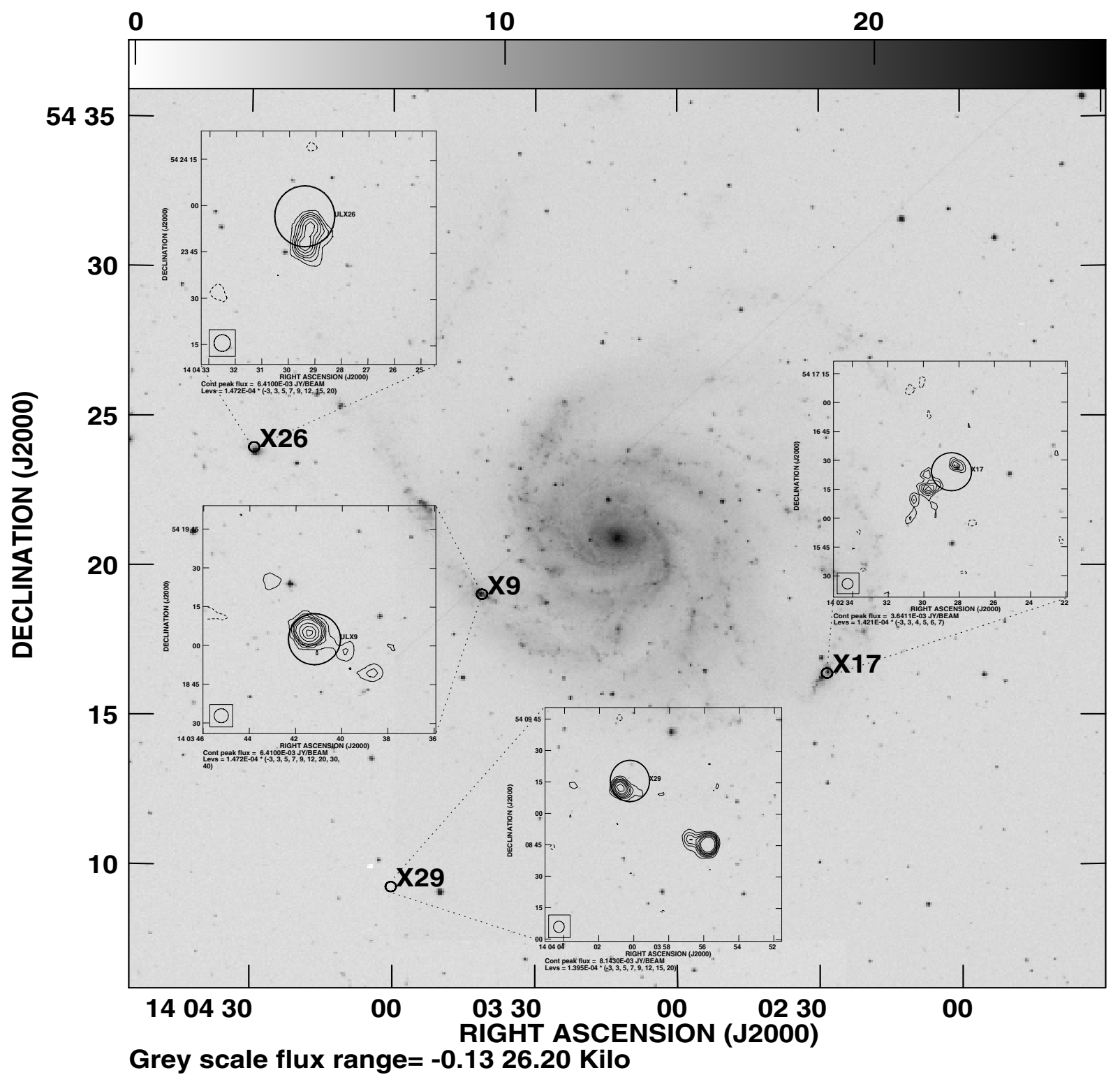

Fig. 8. Several ULX sources (X9, X17, X26, and X29) with new FIRST radio counterpart candidates in the field of NGC 5457 (M 101). 
J. R. Sánchez-Sutil et al.: A catalogue of ULX coincidences with FIRST radio sources (RN), Online Material p 13

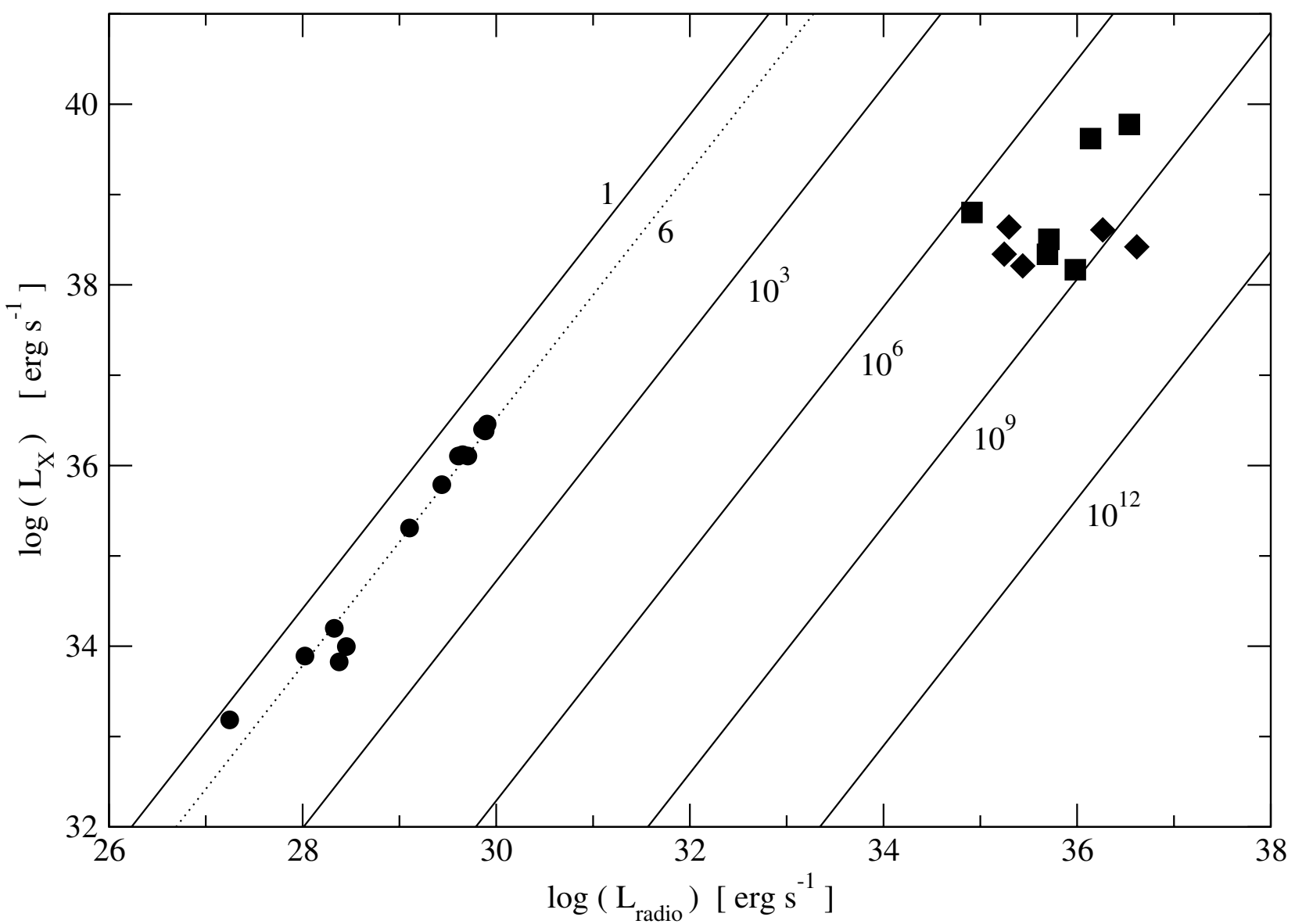

Fig. 9. Comparison of the ULX sources studied with the correlation in the $L_{X}-L_{\text {radio }}$ plane assuming a sub-Eddington radio-loud black-hole scenario. The parallel lines correspond to the labeled black hole mass relative to that of the Sun. The filled circles are the Corbel et al. (2003) data for the $6 M_{\odot}$ black hole X-ray binary GX339-4 where such a correlation is very well established. Diamonds and squares represent peripheral and non-peripheral ULX sources, respectively. 